\title{
A Stage-Structured, Spatially Explicit Migration Model for Myotis Bats: Mortality location affects system dynamics
}

\author{
Richard A. Erickson ${ }^{1, *}$, Wayne E. Thogmartin ${ }^{1}$, Robin E. Russell ${ }^{2}$, \\ Jay E. Diffendorfer ${ }^{3}$, Jennifer A. Szymanski ${ }^{4}$
}

\begin{abstract}
Bats are ecologically and economically important species because they consume insects, transport nutrients, and pollinate flowers. Many species of bats, including those in the Myotis genus, are facing population decline and increased extinction risk. Despite these conservation concerns, few models exist for providing insight into the population dynamics of bats in a spatially explicit context. We developed a model for bats by considering the stage-structured colonial life history of Myotis bats with their annual migration behavior. This model provided insight into network dynamics. We specifically focused on two Myotis species living in the eastern United States: the Indiana bat (M. sodalis), which is a Federally listed endangered species, and the little brown bat (M. lucifugus), which is under consideration for listing as an endangered species. We found that multiple equilibria exist for the local, migratory subpopulations even though the total population was constant. These equilibria suggest the location and magnitude of stressors such as White-nose Syndrome, meteorological phenomena, or impacts of wind turbines on survival influence system dynamics and risk of population extirpation in difficult to predict ways.
\end{abstract}

Keywords: spatial ecology, network dynamics, population biology, Indiana bat, little brown bat

\section{Introduction}

Bats are the second most diverse group of mammals and perform many important ecological and economic roles [28]. These species consume large quantities of insects, benefiting humans [19]. This insect consumption has a significant benefit on important agricultural crops, including forests $[18,35,23]$. The annual economic impact of insect consumption by bats has been estimated at approximately $\$ 23$ billion within the United States [5]. Bats also play an important role in redistributing nutrients across the landscape. For example, the nitrogen from guano piles below the maternity roosts of the northern long-eared bat, Myotis septentrionalis, and Indiana bat, M. sodalis, fertilizes soil and plays a crucial role in forest regeneration and gap dynamics [9]. Despite these important roles, bats are relatively understudied as a taxon and many species are imperiled.

Several Myotis spp. are declining in abundance across eastern North America, including the Federally endangered Indiana bat [1] and the little brown bat, M. lucifugus, a species

\footnotetext{
${ }^{1}$ U.S. Geological Survey, Upper Midwest Environmental Science Center, La Crosse, WI 54603

2 U. S. Geological Survey, National Wildlife Health Center, Madison, WI 53711

3 U.S. Geological Survey, Geosciences and Environmental Change Science Center, Denver, CO 80225

${ }^{4}$ U. S. Fish and Wildlife Service, Division of Endangered Species, Onalaska, WI 54650

${ }^{*}$ Correspondence: rerickson@usgs.gov
} 
being considered for listing under the Endangered Species Act [11]. Both of these species share similar life histories, overwintering in hibernacula and roosting in trees during the summer for breeding, but have differed in their historical abundances. The Indiana bat was one of the first species listed under the Endangered Species Act whereas the little brown bat was the most common bat species in the eastern U.S. until the arrival of the rapidly spreading and novel fungal disease, White-nose Syndrome [10]. In addition to White-nose Syndrome, these species are also facing threats from habitat loss, land use change, wind energy development, and climate change [19, 3].

Despite the ecological and conservation importance of Myotis spp., few models exist to provide insight into their population dynamics (e.g., $[26,10,15,24,32]$ ) and no bat population models were developed until the late 2000s [14]. These models largely focused on winter populations without explicitly considering the movement of bats between caves, shared summer habitat, and the general interconnectedness of bat population dynamics.

Because bats face threats such as land use change (e.g., loss of forested habitat, increase in predation) and wind energy development (e.g., blade mortality and barotrauma) that are route-specific, we wanted to develop a model that included the structure of the migratory network while modeling unique aspects of Myotis biology. To do this, we modified a model developed by Taylor and Norris [30] to include relevant life history traits of Myotis spp. found in the eastern United States.

Taylor and Norris [30] developed a spatially explicit model comprised of complex networks of migratory and non-migratory sites to explore how alterations of populations or their habitat at one site affects other, connected migratory populations. The model by Taylor and Norris [30], constructed with avian taxa in mind, differed from many other frameworks because it focused on the connectivity of migratory populations (e.g., the population moving between points $A$ and $B$ rather than the population at point $A$ ). This detail of their model lends itself to migratory cave bats because the migratory subpopulations face route-specific threats.

We modified the generic, avian model developed by [30] in two ways. We specifically focused on the little brown bat and Indiana bat. First, we included two life-stages rather than a single life-stage. First-year breeding Myotis bats are known to have lower pup production and different survival rates than adults [32]. Second, we relaxed an assumption between the order of arrival at a breeding site and reproductive success. The order or rank of arrival for many bird species determines the quality of habitat available to the animal (e.g., the first bird to arrive secures the best nest site). Conversely, Myotis bats have a colonial breeding structure where arrival order likely plays little social or ecological role [19,3]. We begin by presenting the model structure and then explore how population-level stressors may affect Myotis population dynamics and connectivity.

\section{Model}

\subsection{Model description}

Myotis spp. have a migratory life history that includes multiple life stages (Figures 1 and 2, Tables 2 and 3). The discrete life history of bats lends itself to difference equations with a time step of one year (Equations 1 and 2). Our model reports the number of juveniles and adults during winter because most population monitoring occurs in winter at hibernacula. Both males and females are included as part of the model and this is reflected within the fecundity term. Based upon a previous demographic model [32], the number of first-year breeding bats at the next year $(J(t+1)$; referred to as "juveniles") depends upon four steps: 1) juveniles $(J(t))$ and adults $(A(t))$ from the previous year must survive spring migration $\left(c_{J S}\right.$ and $\left.\left.c_{A S}\right) ; 2\right)$ juveniles and adults must survive the summer $\left(Q_{J}\right.$ and $\left.Q_{A}\right)$; 3 ) juveniles and adults must produce pups $\left(f_{J}\right.$ and $\left.f_{A}\right)$; and finally 4$)$ the pups must survive fall migration $\left(c_{P F}\right)$. 
Brood sites

Hibernacula

(summer)

(winter)

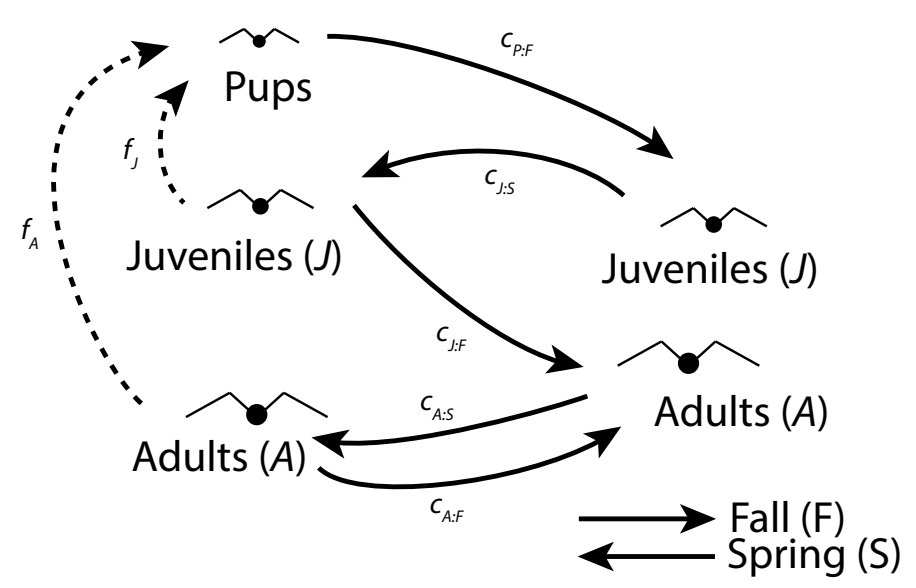

Figure 1: Modeled life cycle of a Myotis spp. that overwinters in a hibernaculum and spends the summer at a breeding site. Parameter names are listed in Table 3. Fall movements are depicted as right arrows and spring movements are depicted as left arrows. The "Pups" include individuals that are born, but have not yet completed their flight to the hibernaculum. "Juveniles" include individuals that have not yet returned to the hibernaculum for their second winter. "Adults" include all other individuals. The dashed lines are births; whereas, the solid lines are movements through time. Parameter names are listed in Tables 1-3.

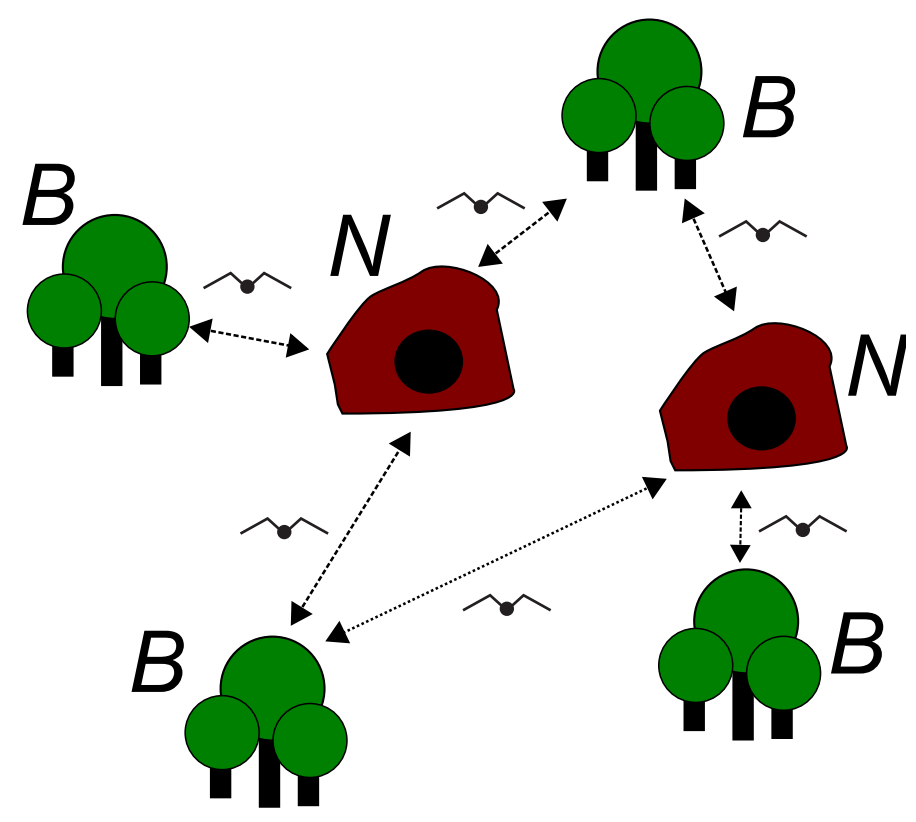

Figure 2: Example of a hypothetical spatial bat population. The $B$ sites are breeding sites and the $N$ sites are non-breeding sites where the bats overwinter. Dashed lines indicate migratory pathways. 
Adult dynamics involve three steps: 1) the pups become juveniles after surviving the fall migration (which is at about 3-4 months of age); 2) the juveniles become adults after surviving their first winter $\left(V_{J}\right)$, and then survive the spring migration $\left(c_{S J}\right)$, summer $\left(Q_{J}\right)$, and fall migration $\left(c_{F J}\right)$ (which is at about 15-16 months of age); and 3) Adults migrate during the fall $\left(c_{A F}\right)$ to the hibernacula where they overwinter $\left(V_{A}\right)$ and then migrate during the spring $\left(c_{S A}\right)$ to breeding sites where they spend the summer $\left(Q_{A}\right)$. This cycle continues until the adult bats die.

Myotis populations are connected [29], with members of a summer roost site originating from different hibernacula [21]. To allow the model to track subpopulations using different migration routes, we incorporated spatial dynamics into the model following the approach used by Taylor and Norris [30]. Our parameter names differed from [30] reflecting differences between avian and bat ecology; we have included a table that crosswalks both their names and ours (Table 1). Each subpopulation has a unique migration route between non-breeding $(n \in 1,2,3, \ldots, N)$ and breeding site $(b \in 1,2,3, \ldots, B)$. This spatially explicit form of the model we used becomes the following set of equations:

$$
\begin{aligned}
J_{n, b}(t+1)= & c_{P: n, b: F} c_{J: n, b: S} f_{J: n, b} Q_{J: n}(t) J_{n, b}(t) \\
& +c_{P: n, b: F} c_{A: n, b: S} f_{A: n, b} Q_{A: n}(t) A_{n, b}(t), \\
A_{n, b}(t+1)= & c_{J: n, b: S} c_{J: n, b: F} Q_{J: b}(t) V_{J: n}(t) J_{n, b}(t) \\
& +c_{A: n, b: S} c_{A: n, b: F} Q_{A: b}(t) V_{A: n}(t) A_{n, b}(t) .
\end{aligned}
$$

Table 1: Parameter symbols used by Taylor and Norris [30] compared to symbols and names used within our model. We separated out the $F$ matrix to be a separate fecundity term $f$ and breeding site carrying capacity $V$. For simplicity, we include neither the subscripts that indicate age structure within our model nor most site-specific subscripts. It is also worth noting that some, but not all, of our matrices are transposed from the matrices of Taylor and Norris [30].

\begin{tabular}{ccl}
$\begin{array}{c}\text { Taylor and Norris } \\
\text { symbol [30] }\end{array}$ & $\begin{array}{c}\text { our } \\
\text { symbol }\end{array}$ & Parameter value \\
\hline$N_{B}$ & $B$ & Number of breeding sites \\
$N_{W}$ & $N$ & Number of non-breeding (winter) sites \\
$D_{i j}$ & $D_{n, b}$ & Distance between sites \\
$c_{i j}$ & $c_{n, b}$ & Migration survival \\
$d^{\prime}$ & - & Site rank (removed) \\
$b^{\prime}$ & - & Site rank (removed) \\
$d$ & $v$ & Non-breeding site baseline survival fecundity \\
$q$ & $q$ and $f$ & Breeding site baseline survival and fecity \\
$K_{b}$ & $K_{b}$ & Breeding site carrying carrying capacity \\
$K_{n}$ & $K_{n}$ & Non-breeding site carrying carrying capacity \\
$F$ & $Q$ & Breeding site survival matrix \\
$S$ & $V$ & Non-breeding site survival matrix \\
\hline
\end{tabular}

As an example of our subscript syntax, the adult subpopulation migrating between non-breeding site 1 and breeding site 3 would be $A_{13}$ while total number of individuals overwintering at non-breeding site 1 would be the sum of all individuals migrating from any breeding site to site 1 (i.e., $\sum_{b=1}^{B} A_{1 b}$ ). The migration survival $(c)$ between sites affects subpopulation size and is a function of distance (e.g., $c_{J: n, b: S}$ refers to the subpopulation that overwinter at non-breeding $n$ and spends the summer at breeding site $b$, rather than migrating from site $n$ to site $b$ ). This is in agreement with previous observations of increasing mortality associated with increasing migration distance [16]. We assumed a constant migration survival rate across all life stages, but this parameter could be modified if data 
existed indicating survival rate differences among stages (Equations $3-7$ ). Furthermore, this term can have additional migration mortality $(\mu)$ caused by outside stressors, such as mortality from meteorological phenomena or wind turbines, that might vary between spring and fall migrations [3]. Accordingly, survival is

$$
\begin{aligned}
& c_{J: n, b: S}=\left(1-\mu_{s}\right) e^{\left(-0.01 D_{n, b}\right)}, \\
& c_{A: n, b: S}=\left(1-\mu_{s}\right) e^{\left(-0.01 D_{n, b}\right)}, \\
& c_{P: n, b: F}=\left(1-\mu_{f}\right) e^{\left(-0.01 D_{n, b}\right)}, \\
& c_{J: n, b: F}=\left(1-\mu_{f}\right) e^{\left(-0.01 D_{n, b}\right)}, \\
& c_{A: n, b: F}=\left(1-\mu_{f}\right) e^{\left(-0.01 D_{n, b}\right)} .
\end{aligned}
$$

A density-dependent function is used to model survival in each non-breeding site (Equations 8-9) and breeding site (Equations 10-11). These functions, following directly from Taylor and Norris [30], reflect not only the amount of physical space for bats in caves or other roost sites, but also the amount of feeding habitat surrounding roosts. Note that the survival at the summer sites $(\mathrm{Q})$ sum over the different non-breeding groups $\left(b_{1}, b_{2}, \ldots, b_{B}\right)$ that use the summer sites:

$$
\begin{aligned}
& Q_{J: b}(t)=q_{j} e^{\sum_{n=1}^{N}-\frac{A_{n, b}(t)+J_{n, b}(t)}{K_{B b}}}, \\
& Q_{A: b}(t)=q_{a} e^{\sum_{n=1}^{N}-\frac{A_{n, b}(t)+J_{n, b}(t)}{K_{B b}}}, \\
& V_{J: n}(t)=v_{j} e^{\sum_{b=1}^{B}-\frac{A_{n, b}(t)+J_{n, b}(t)}{K_{N n}}}, \\
& V_{A: n}(t)=v_{a} e^{\sum_{b=1}^{B}-\frac{A_{n, b}(t)+J_{n, b}(t)}{K_{N n}}} .
\end{aligned}
$$

Each breeding site has a site-specific fecundity for both juveniles and adults. This term includes pup production (i.e., number of births) as well as the probability of surviving to become juveniles. The maximum fecundity is 0.5 because our species of Myotis only produces a maximum of 1 pup per year and we assume that half the population are males who do not give birth $[3,32]$. This is in contrast to some other species of bats capable of producing multiple pups per season [3].

\section{$2.2 \quad$ Parameter values}

Parameter values were based upon those found within the literature for an assortment of Myotis spp. (Tables 2-3). Each hibernaculum and each summer roost site has its own carrying capacity. This models a system of hibernacula and summer roost sites [8]. Following the assumptions of Taylor and Norris [30], our model included density at both the hibernacula and summer roost sites. This assumption was retained to maintain consistency with the previous model. The fecundity rate reflects that younger bats are smaller and are less likely to be successful at producing pups [3], assumes a 1:1 sex ratio [3], and a relatively low mortality rate is incurred by pups during non-migratory periods [3]. The overall annual survival rate of juveniles was assumed to be lower than adults (about $60 \%$ vs. about $95 \%$ ). These rates were calculated by multiplying the baseline seasonal survival parameters and corresponds to a maximum life expectancy of about twenty years, which commonly occurs with Myotis spp. [3]. The high seasonal parameter choice is also in agreement with modeling efforts by others demonstrating that the overwinter survival is likely $>0.96$ [4].

The "cost" of migration (decrease in survival as a function of distance) was modeled using an exponential decay function (Equations 3-7) [30]. This function reflects that bats endure a migration cost and decreases the size of subpopulations migrating a longer distance [8]. Our model currently assumes all three life stages (pups, juveniles, and adults) have the same migration limits and that both spring and fall migration distances are the same. The 
Table 2: State parameter and life stages of Myotis bats in this model. The subscripts refer to the individual non-breeding sites, $n$, and breeding site, $b$, to which a bat subpopulation migrates.

\begin{tabular}{lcl} 
Life stage & Parameter & Bat age \\
\hline Pup & - & Through the first fall migration \\
Juveniles & $J_{n, b}$ & Have not yet completed their second fall migration cycle \\
Adult & $A_{n, b}$ & Have completed their second fall migration \\
\hline
\end{tabular}

Table 3: Life parameters of Myotis bats in this model. Some parameters have subscripts that refer to the individual non-breeding sites, $n$, or breeding site, $b$. Additionally, some parameters have a subscript that refers to either a juvenile, $J$, or an adult, $A$, subpopulation, and one parameter has a $P$ that refers to pups. The listed parameter values are used in all simulations unless otherwise noted. Additionally, a subscript may be used to denote spring, $S$, or fall, $F$, for migration related parameters. Note that subscripts on migration parameters refer to the subpopulation, not the direction of travel (e.g., $c_{J: n, b: S}$ refers to the subpopulation that overwinters at non-breeding site $n$ and spends the summer at breeding site $b$, rather than migrating from site $n$ to site $b$ ).

\begin{tabular}{|c|c|c|}
\hline $\begin{array}{l}\text { Parameter } \\
\text { symbol }\end{array}$ & Parameter name & $\begin{array}{l}\text { Parameter value } \\
\text { or equation }\end{array}$ \\
\hline$f_{J: b}$ & Juvenile fecundity rate for breeding site $b$ & $0.25 \forall b, b \in 1,2, \ldots, B$ \\
\hline$f_{A: b}$ & Adult fecundity rate for breeding site $b$ & $0.45 \forall b, b \in 1,2, \ldots, B$ \\
\hline$D_{n, b}$ & $\begin{array}{l}\text { Distance between non-breeding site } n \text { and } \\
\text { breeding site } b\end{array}$ & (input parameter, see text) \\
\hline$c_{J: n, b: S}$ & Spring juvenile migration survival & Equation 3 \\
\hline$c_{A: n, b: S}$ & Spring adult migration survival & Equation 4 \\
\hline$c_{P: n, b: F}$ & Fall pup migration survival & Equation 5 \\
\hline$c_{J: n, b: F}$ & Fall juvenile migration survival & Equation 6 \\
\hline$c_{A: n, b: F}$ & Fall adult migration survival & Equation 7 \\
\hline$q_{J: b}$ & Juvenile survival rate for breeding site $b$ & $0.80 \forall b, b \in 1,2, \ldots, B$ \\
\hline$q_{A: b}$ & Adult survival rate for breeding site $b$ & $0.975 \forall b, b \in 1,2, \ldots, B$ \\
\hline$v_{J: n}$ & $\begin{array}{l}\text { Juvenile overwinter survival at non- } \\
\text { breeding site } n\end{array}$ & $0.80 \forall n, n \in 1,2, \ldots, N$ \\
\hline$v_{A: n}$ & $\begin{array}{l}\text { Adult overwinter survival at non- } \\
\text { breeding } n\end{array}$ & $0.975 \forall n, n \in 1,2, \ldots, N$ \\
\hline$K_{B b}$ & Carrying capacity for breeding site $b$ & $K_{B b} \in[10,000,60,000]$ \\
\hline$K_{N n}$ & $\begin{array}{l}\text { Carrying capacity for hibernaculum site } \\
n\end{array}$ & $K_{N n} \in[100,000,600,000]$ \\
\hline$Q_{J: b}(t)$ & Juvenile breeding site survival & Equation 8 \\
\hline$Q_{A: b}(t)$ & Adult breeding site survival & Equation 9 \\
\hline$V_{J: n}(t)$ & Juvenile non-breeding site survival & Equation 10 \\
\hline$V_{A: n}(t)$ & Adult non-breeding site survival & Equation 11 \\
\hline
\end{tabular}




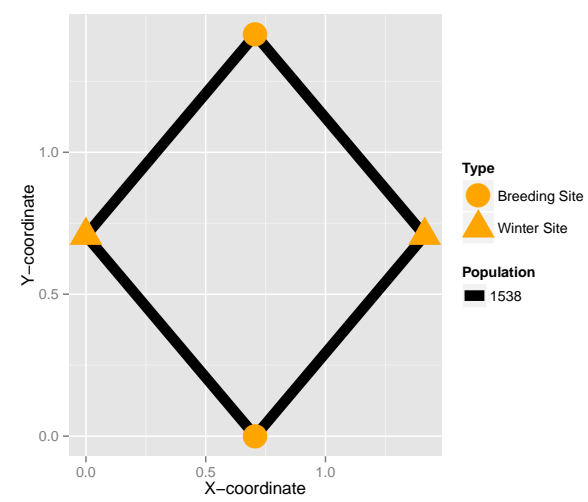

(a) Identical initial conditions.

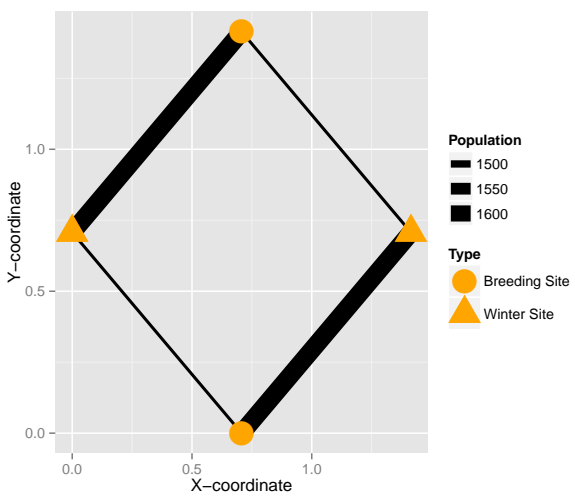

(b) Different initial conditions.

Figure 3: Site locations and final migratory subpopulation sizes for a simple migratory simulation. The site shape indicates the type of site. The width of the line indicates the final migratory subpopulation size.

migration function we used is parameterized to the same arbitrary unit of 1 used by Taylor and Norris [30]. This parameter can easily be scaled for the population and area of interest.

\section{$2.3 \quad$ Numerical methods and simulations}

We presented two different spatial landscapes to demonstrate and explore our model: a 'simple' landscape and a 'complex' landscape. With these landscapes, we explored the effects of varying carrying capacities, distances between hibernacula and roosts, stability, and varying amounts of "take" on the model's dynamics.

The simple landscape was designed to allow in-depth simulation studies. It consisted of two non-breeding sites and two breeding sites (Figure 3), in a diamond shape with breeding and winter sites adjacent to each other, so that all migration was along the equidistant perimeter paths, with a fixed distance of 1 unit. The density term, $K$, was 30,000 individuals at each breeding site and hibernaculum (i.e., $K_{B 1}=K_{B 2}=K_{N 1}=K_{N 2}=30,000$ ) controlling for carrying capacity. Taylor and Norris [30] noted the model should produce "multiple unstable equilibria and (usually) one stable." Based upon preliminary model exploration, we observed multiple equilibria that appeared to be attractors and depended upon the initial conditions of the model (i.e., the route-specific populations). These equilibria numerically converged, but small perturbations to the system sometimes caused the model to find different equilibria. Based upon these observations, we ran simulations with 1,490 different sets of initial conditions to explore the model space. This set included empty subpopulations (i.e., one or more routes without any individuals). These initial conditions also could be thought of as a perturbation analysis, which is one method of analyzing local stability [7]. For this analysis, 205 years of simulations were run although only the first 100 were plotted to allow visualization of the transient dynamics.

We also analyzed the effects of distance on population size by "moving" one migratory population. For this, we changed the migration distance for one breeding site compared to both hibernacula while keeping the other breeding location constant. The new distances were $0.5,1,2,4$, and 8 units. This provided insight into the effects of distance on spatial system dynamics.

The second experimental landscape was a complicated landscape lacking the symmetry of the simple landscape. This landscape was simpler than the spatial array of little brown bat complexes (e.g., [29]), but approaches biological realism while still maintaining tractability. This landscape included three non-breeding and ten breeding sites (Figure 6, Table 4). The initial subpopulation was comprised of 2,000 adults and 1,000 juveniles for every migration 
route. Different density-dependent or carrying capacity terms were used at the different sites (Table 4). A second set of simulations was run with only two carrying capacities, one for all breeding sites, and another for all non-breeding sites. We ran the complex landscape simulations 6,000 years to ensure convergence was reached. This was required because a long time period was required for some subpopulations to go extinct. Specifically, some populations required a long time to converge numerically to zero.

Table 4: Coordinates for non-breeding sites and breeding sites for the more complicated model. The coordinates are on an arbitrary distance scale.

\begin{tabular}{lccc} 
Site name & $x$-coordinate & $y$-coordinate & carrying capacity \\
\hline Non-breeding 1 & 6 & 7 & 100,000 \\
Non-breeding 2 & 4 & -4 & 200,000 \\
Non-breeding 3 & -2 & -2 & 200,000 \\
Non-breeding 1 & 2 & -3 & 10,000 \\
Breeding 2 & 4 & 4 & 40,000 \\
Breeding 3 & -6 & 3 & 60,000 \\
Breeding 4 & 15 & 7 & 10,000 \\
Breeding 5 & -1 & -5 & 20,000 \\
Breeding 6 & 0 & 2 & 30,000 \\
Breeding 7 & -4 & -7 & 10,000 \\
Breeding 8 & 8 & -2 & 10,000 \\
Breeding 9 & -8 & 8 & 10,000 \\
Breeding 10 & -2 & 6 & 10,000 \\
\hline
\end{tabular}

Finally, we ran a set of simulations using both landscapes to investigate simulated mortality from wind farms. We only considered lethal take within our model. Homogeneous conditions (e.g., uniform carrying capacities across sites and parameter values) were used for both landscapes. Equilibrium population values were used for the initial population sizes for the complex landscape and populations slightly above equilibrium values were used for the diamond pattern landscape.

Four scenarios were run on the simple, diamond pattern landscape: 1) a reference simulation with no additional migration mortality; 2) a simulation with $2 \%$ take of populations migrating to the second breeding site during both spring and fall; 3) a simulation with $10 \%$ take of populations migrating to the second breeding site during both spring and fall; and 4) a simulation with $10 \%$ take of populations migrating between breeding site 2 and non-breeding site 2. The second and third scenarios might represent the development of a wind farm near a breeding site affecting all migrants to and from that site. The fourth scenario might represent a wind farm development along a single migration route.

Three scenarios were run for the complex landscape: 1) a reference simulation; 2) a simulation with $10 \%$ take during both spring and fall migrations affecting breeding site 1 ; and 3) a simulation with $2 \%$ take during both spring and fall migration affecting the third hibernaculum. The second simulation might represent a wind farm development that only affects one subpopulation. The third simulation might represent a wind farm development affecting one hibernaculum.

All of our numerical simulations were conducted using $\mathrm{R}$ [25] and we have included several functions as part of our online supplemental. The main function Bat_Mig runs the population simulation through time. The function Site_Order produces the order in which coordinates and other site information should be entered into the function. This function is useful because we used vectors rather than matrices for the numerical implementation of our model. The function Site_Dist calculates the migration distances. The function Site_Plot plots the sites and the function Site_Plot_Mig plots both the final migration subpopulation sizes and site locations. Figures were plotted using the ggplot2 package [33]. 


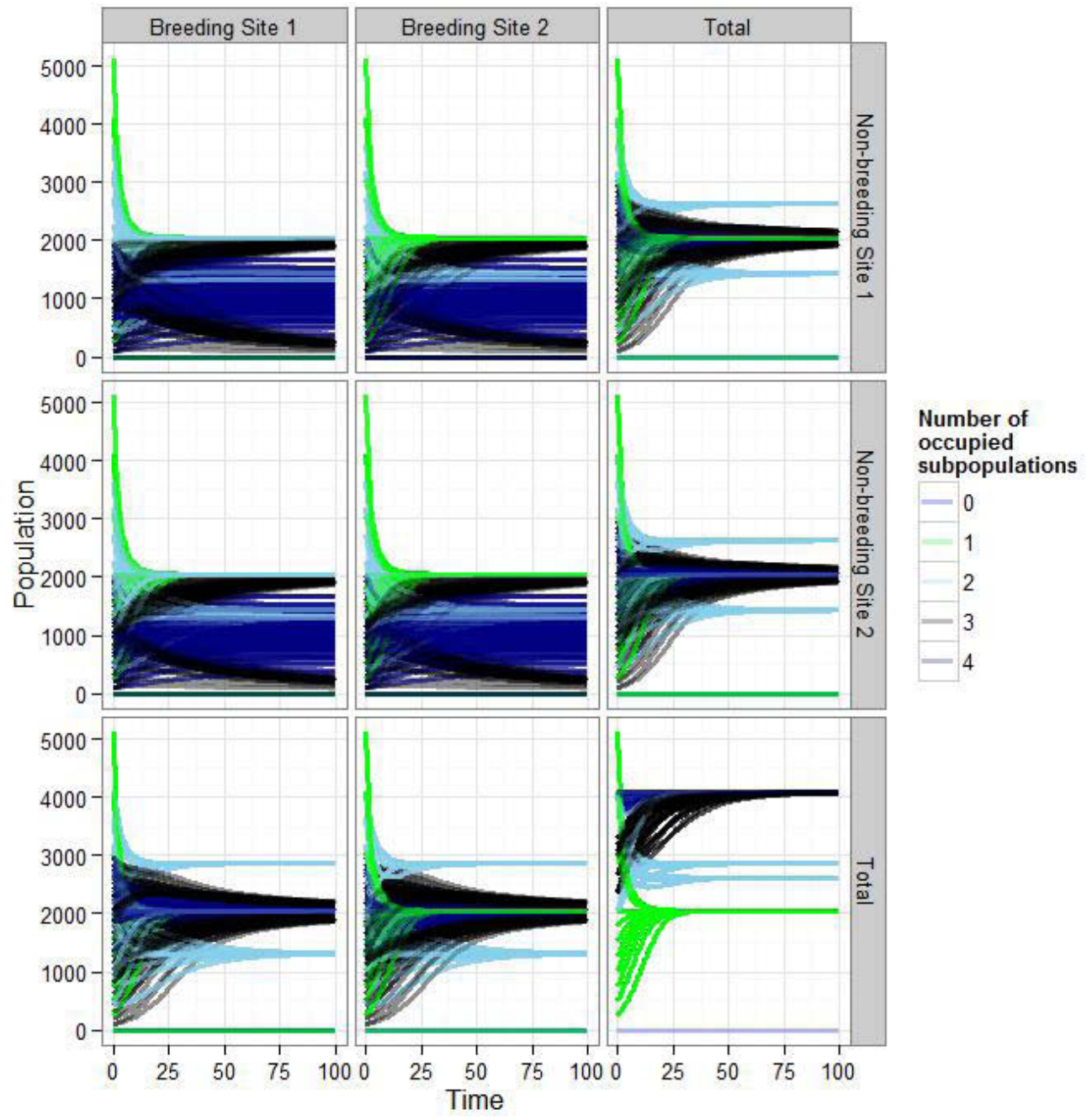

Figure 4: Results of 1,490 different initial conditions. The different colors represent different numbers of initial occupied sites. For example, if three subpopulations were present, one migratory route would be unused.

\section{Results}

\subsection{Simple landscape results}

Regardless of the initial conditions, populations always reached equilibria without any multistate cycling occurring (Figure 4). Initial conditions, perturbations away from the equilibria values, the number of occupied sites, and the location of occupied sites in the migratory system all affected model behavior, the total population size, and the final population size. The single-occupied subpopulation simulations always reached a single equilibrium value as did the total populations when 1, 3, and 4 subpopulations were occupied. The twooccupied subpopulation simulations had three different outcomes depending upon which sites were occupied: two subpopulations "parallel" to each other and not sharing any sites, two-subpopulations sharing a hibernaculum, or two-subpopulations sharing a breeding site. Each of the configurations also led to a different total population size. The three-occupied subpopulation simulations had two different equilibria values for each subpopulation and this corresponded to which migratory populations were not sharing either a breeding or 


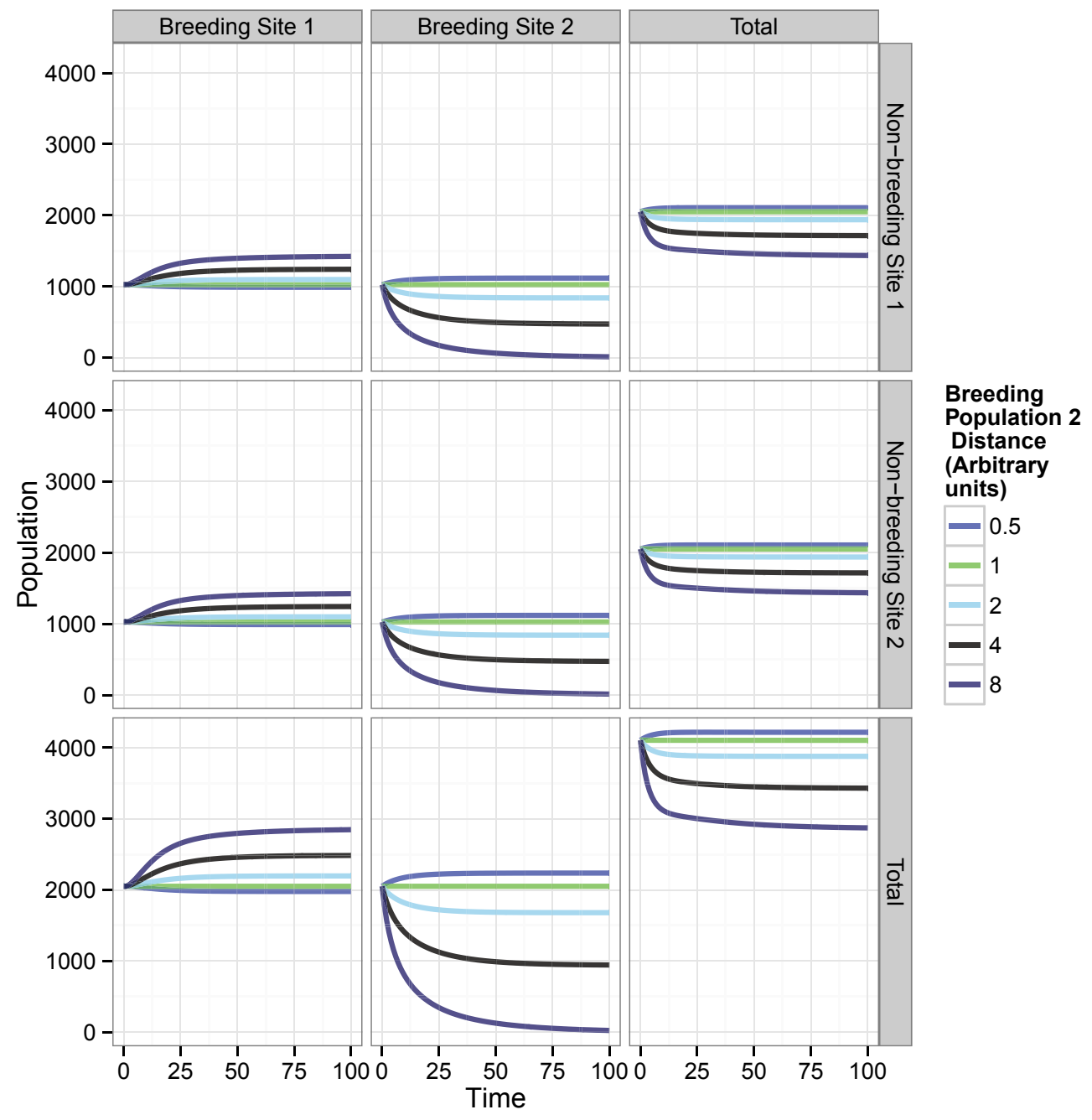

Figure 5: Results of changing the distance between breeding site 2 and the non-breeding sites.

non-breeding site. The three subpopulation simulations always had the same total population size at equilibrium. When three subpopulations were present, the subpopulation sharing both the breeding and non-breeding site decreased to $\sim 10$ bats, but did not proceed to extirpation. The four occupied subpopulation simulations had many different equilibria for the individual subpopulations, but the total population always converged to the same equilibrium value. Transient dynamics appeared to be less important for the four subpopulation model compared to the other models. For all scenarios, population size became static within the system once the total carrying capacity of the system was reached.

Changing the distance between a breeding site and non-breeding site changed the size of the subpopulation in a manner consistent with a cost associated with migration distance. Farther distances had lower population sizes along migratory routes, while shorter distances had larger population sizes (Figure 5). Additionally, the systems with shorter migration distances had greater total population sizes, ostensibly because of reduced migration mortality. 


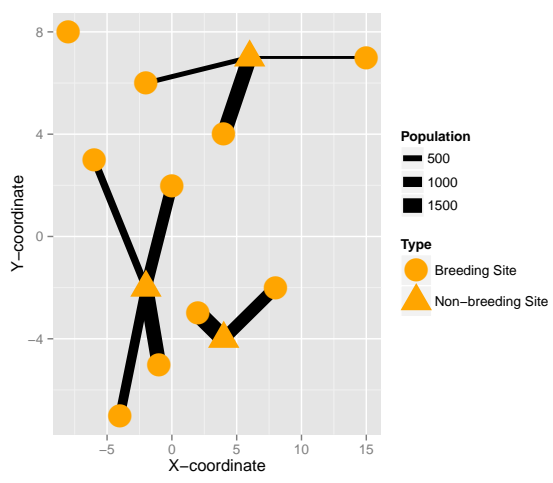

(a) Equal carrying capacities.

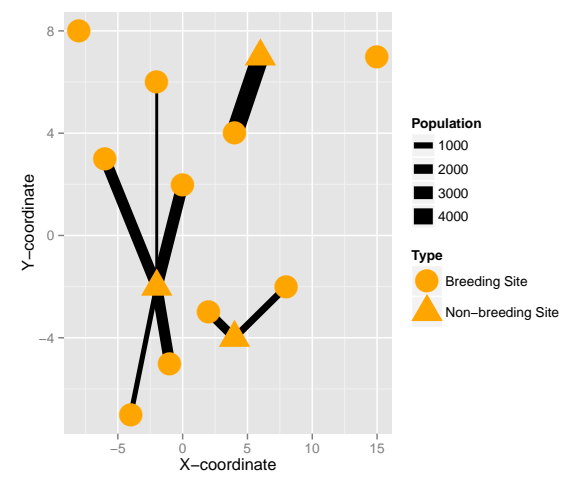

(b) Unequal carrying capacities.

Figure 6: Site locations and final migratory population sizes for a complex migratory simulation. The site shape indicates the type of site. The width of the line indicates the final migratory population size.

\subsection{Complex landscape simulations}

The more complex spatially structured population produced heterogeneous subpopulation sizes at equilibrium (Figure 6). This included multiple sites being left unoccupied because of the distance between hibernacula and breeding sites. The noticeable difference between Figure $6 \mathrm{a}$ and Figure $6 \mathrm{~b}$ describes the importance of both distance and site-specific carrying capacity.

\subsection{Simulating impacts of wind energy}

Increased migration mortality changed the population dynamics (Figures 7 and 8 ) in a manner dictated by density dependency and the common use of non-breeding sites. The $2 \%$ take scenario decreased the subpopulation using the second breeding site as would be expected from increased mortality. This decline in the subpopulation using the second breeding site caused a slight increase in abundance of the populations using the first breeding site because both subpopulations were regulated by density dependency at non-breeding sites, which they shared. The mortality in the subpopulation allowed the other subpopulation to grow until the combined abundance of both subpopulations reached the carrying capacity of the non-breeding sites.

However, the increase in the subpopulation not facing added mortality was not enough to offset the loss from mortality, and led to a net decrease in the system-wide population size. The 10\%-take migration produced similar results but caused the extirpation of populations using the second breeding site. The 10\%-take of the population migrating between breeding site 2 and the non-breeding site caused extirpation of this subpopulation; this scenario also caused an increase in the two subpopulations sharing either the non-breeding or breeding site. The increases in these two subpopulations then caused a decrease in the subpopulation "parallel" to the originally affected subpopulation. This increase occurred because the the two subpopulations sharing sites with this population experienced a release from the consequences of density. The increase is not necessarily intuitive and is an artifact of the model that may or may not be representative of reality.

The effects of increased migration mortality on the complex landscape system were more straightforward. The $10 \%$ migration take that only affected one population caused extirpation of that population. The $2 \%$ migration take affecting the fifth breeding site caused small decreases in these populations. Unexpectedly, both scenarios had similar decreases on the total population size, indicating density-dependent compensation with increasing mortality from take. 


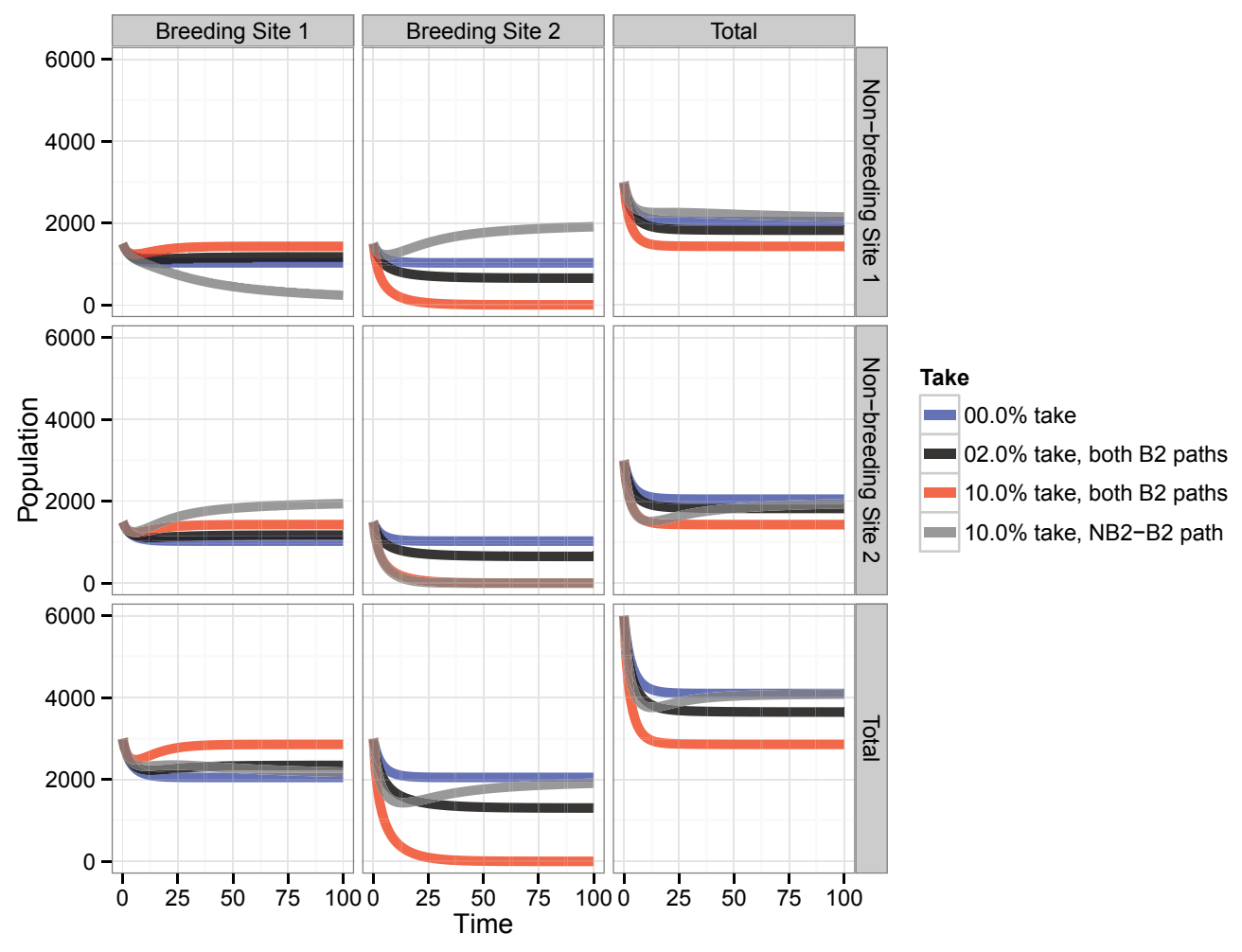

Figure 7: Effects of increasing migration mortality on different subpopulations. The label "both B2 paths" refers to the subpopulations that use the second breeding site, while "NB2-B2 path" refers to the subpopulation that migrates between the second breeding site and second non-breeding site.

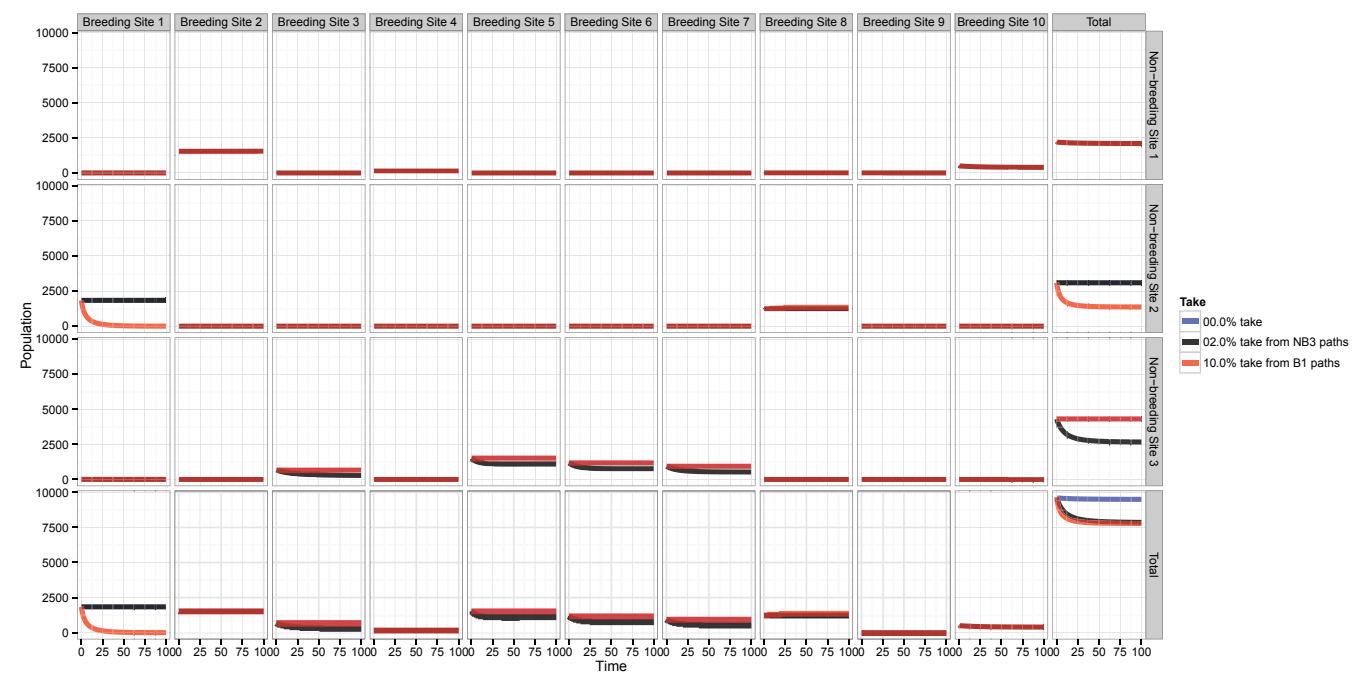

Figure 8: Effects of increasing migration mortality on different subpopulations. The label "take from NB3 paths" refers to the subpopulations that use the third non-breeding site, while "B1 path" refers to the subpopulations that use the first breeding site. 


\section{Discussion}

Our model provides insight into both the population dynamics of bats and the more general spatial dynamics of migratory species by considering the effects of a multi-life staged organism aggregating in colonies. Our most surprising result was the presence of multiple equilibria depending upon initial conditions or perturbations. This finding differed from previous modeling efforts using a similarly structured modeling approach [30, 34]. Overall, our system-wide dynamics were stable, but the presence of multiple equilibria leads to important ramifications for the system from both a numerical and biological perspective. For example, numerical parameterizations of the model using optimization methods would likely only yield one solution to the problem; this solution would be dependent upon the numerical method used for parameterization as well as the initial values of the parameterizations [2]. Similarly, a Bayesian Markov Chain Monte Carlo method may find multiple conditions depending upon the prior distributions used, which may lead to poor numerical convergence [12]. Biologically, multiple equilibria may lead to multiple steady states for the system. The implications of this result are that disturbance may permanently alter the system to a new state, one inflexible to management action [27].

We found the transient dynamics of the system largely dissipated after 25 years for all but the largest perturbations (or initial conditions farthest away from the equilibrium). Given existing rates of change in bat habitats, it may be difficult for the species modeled here to achieve stability, because they may be perturbed more frequently than every 25 years. The assumption of a static environment may be a biologically unreasonable outcome because system changes would likely occur during this time period and the underlying assumption of a static environment is rarely appropriate. Additionally, some subpopulations would either over or undershoot the equilibrium value before settling down. However, we observed no oscillation and no multi-cycle solution appeared with our parameter choices. This result occurs because the model includes density functions for limiting the upper size of populations.

As expected, increasing distance between populations decreased subpopulation size. This decrease resulted in an increase in other subpopulation sizes, though the net result was a decrease in total population size. This consequence of distance was exhibited in the more complex landscape example, with more distant sites having smaller populations or being unoccupied. This finding was not surprising, but lends realism to the model. Despite the effects of distance and carrying capacity on population dynamics, both our simple and complex experimental systems had sites used by two or more different migratory subpopulations. This result was not reported by Wiederholt et al. [34] who found only one subpopulation per site. However, Myotis spp. have been observed sharing both summer roost sites and hibernacula with bats from other sites (e.g., two bats at a summer roost need not share a hibernaculum) [21]. One possible reason for this difference may be that Wiederholt et al. [34] included the rank function that we removed from the model. Including the rank function penalizes populations migrating long distances and decreases the opportunity for co-existence of populations originating from different locations.

The multiple equilibria, transient dynamics, and effects of distance may affect the conservation of bats or similar colonially roosting species. A small disturbance at a single site will likely be offset to some extent by an increase in other subpopulations through densitydependent processes. A larger disturbance though may allow some populations to increase in size even though the majority of populations and the total overall population are decreasing. The arrival of White-nose Syndrome in the eastern United States caused a large die off of cave bats, with winter bat populations decreasing in size [32]. Nevertheless, some populations increased in the region, possibly as a result of bats emigrating from the area of infection [31].

The transient dynamics we observed also suggest a population may take a long time to recover from a major disturbance. This is important for conservation of endangered species such as the Indiana bat because the U.S. Fish and Wildlife Service issues "take" 
permits under the Endangered Species Act of 1973 and these permits allow individuals to be killed or otherwise disturbed by activities such as energy production or development. If great enough, this disturbance may result in a permanent shift in population structure or recovery from this disturbance may require a greater time period than expected. Changing the distance between populations may become important as well for bats. Loss of summer habitat may change the distance subpopulations are required to fly during migration, which in turn shifts hibernacula dynamics through its effects on density dependence.

The take scenarios also illustrate the importance of spatial dynamics. The diamond shaped or simple landscape scenarios illustrated the need for considering nonlinear dynamics and connections between populations. The complex landscape illustrated how similar declines in the total population have very different metapopulation implications. One scenario caused the extirpation of individuals at a site, but the other scenario caused small and barely observable decreases to subpopulations. This difference illustrates how not all population loss is the same across a landscape with different subpopulations. In metapopulation models, the loss of subpopulations may increase the risk of extinction for the entire metapopulation [13]. Though we did not explicitly study risk of system-wide extinction with our model, our results suggest the loss of a single migratory subpopulation or site (either breeding or overwinter) can alter the total population size and the spatial dynamics. Thus, resource managers need to consider incremental population loss similar to incremental habitat loss [6] and may need to understand the location of loss for correctly characterizing risk to the system.

Our model works best when dealing with large populations not subject to stochasticity. Bat populations, especially at summer sites, are often small and demographic stochasticity is important for populations of this size [22]. The model as formulated also assumes strict site fidelity without colonization of new locations. This assumption is valid for short time scales because, for example, bats exhibit strong site attachment to specific summer roost sites [17]. Over longer periods of time, these species require behavioral flexibility to change summer breeding sites because of the transient nature of roost habitat [17]. Additional research has shown that Myotis bat populations will colonize new sites, especially winter sites [20]. Relaxing these assumptions would require the inclusion of stochastic demographic and movement parameters. This extra cost in complexity though would increase the utility of this framework within a decision making context.

\section{Acknowledgment}

Any use of trade, product, or firm names are for descriptive purposes only and do not imply endorsement by the U.S. Government. The views expressed in this article are the authors' own and do not necessarily represent the views of the U.S. Fish and Wildlife Service.

\section{References}

[1] 32FR4001. Notices. Federal Register, 32:48:4001, March 11, 1967.

[2] A. S. Ackleh, E. J. Allen, R. B. Kearfott, and P. Seshaiyer. Classical and Modern Numerical Analysis: Theory, Methods and Practice. CRC Press, Boca Raton, 2011.

[3] J. D. Altringham. Bats: From Evolution to Conservation. Oxford, New York, 2011.

[4] J. G. Boyles and V. Brack, Jr. Modeling survival rates of hibernating mammals with individual-based models of energy expenditure. Journal of Mammalogy, 90:9-16, 2009.

[5] J. G. Boyles, P. M. Cryan, G. F. McCracken, and T. H. Kunz. Economic importance of bats in agriculture. Sciences, 332:41-42, 2011. 
[6] T. M. Brooks, R. A. Mittermeier, C. G. Mittermeier, G. A. B. Da Fonseca, A. B. Rylands, W.R Konstant, P. Flick, J. Pilgrim, S. Oldfield, G. Magin, and C. HiltonTaylor. Habitat loss and extinction in the hotspots of biodiversity. Conservation biology, 16:909-923, 2002.

[7] H. Caswell. Matrix Populations Models. Sinauer Associates, Sunderland, Massachusetts, USA, 2001.

[8] W. H. Davis and H. B. Hitchcock. Biology and migration of the bat, Myotis lucifugus, in New England. Journal of Mammalogy, 46:296-313, 1965.

[9] J.E. Duchamp, D. W. Sparks, and R. K. Swihart. Exploring the "nutrient hot spot" hypothesis at trees used by bats. Journal of Mammalogy, 91:48-53, 2010.

[10] W. F. Frick, J. F. Pollock, A. C. Hicks, K. E. Langwig, D. S. Reynolds, G. G. Turner, C. M. Butchkoski, and T.H. Kunz. An emerging disease causes regional population collapse of a common north american bat species. Science, 329:679-682, 2010.

[11] A. Froschauer, M. Racey, and C. Riley. Review finds engangered species protection may be warranted for two bat species. Technical report, U. S. Fish and Wildlife Service, 2011.

[12] A. Gelman, J. B. Carlin, H. S. Stern, B.D. Dunson, A. Vehtari, and D. B. Rubin. Bayesian Data Analysis. CRC press, Boca Raton, FL, 2013.

[13] N. J. Gotelli. A Primer of Ecology. Sinauer, Sunderland, fourth edition, 2008.

[14] T. G. Hallam and P. Federico. Ecological and Behavioral Methods for the Study of Bats, chapter Application of Dynamic Population Models to Bats, pages 177-194. Johns Hopkins University Press, Baltimore, 2009.

[15] T. G. Hallam and G. F. McCracken. Management of the panzootic white-nose syndrome through culling of bats. Conservation Biology, 25:189-194, 2011.

[16] S. R. Humphrey and J. B. Cope. Survival rates of the endangered Indiana bat, Myotis sodalis. Journal of Mammalogy, pages 32-36, 1977.

[17] S. R. Humphrey, A. R. Richter, and J. B. Cope. Summer habitat and ecology of the endangered Indiana bat, Myotis sodalis. Journal of Mammalogy, pages 334-346, 1977.

[18] M. B. Kalka, A.R. Smith, and E. K. V. Kalko. Bats limit arthropods and herbivory in a tropical forest. Science, 320:71, 2008.

[19] T. H. Kunz, E. Braun de Torrez, D. Bauer, T. Lobova, and T. H. Fleming. Ecosystem services provided by bats. Annals of the New York Academy of Sciences, 1223:1-38, 2011.

[20] A. Kurta, D. King, J. A. Teramino, J. M. Stribley, and K. J. Williams. Summer roosts of the endangered Indiana bat (Myotis sodalis) on the northern edge of its range. American Midland Naturalist, pages 132-138, 1993.

[21] A. Kurta and S. W. Murray. Philopatry and migration of banded Indiana bats (Myotis sodalis) and effects of radio transmitters. Journal of Mammalogy, 83:585-589, 2002.

[22] B. A. Melbourne and A. Hastings. Extinction risk depends strongly on factors contributing to stochasticity. Nature, 454:100-103, 2008.

[23] E. B. Morrison and C. A. Lindell. Birds and bats reduce insect biomass and leaf damage in tropical forest restoration sites. Ecological Applications, 22:1526-1534, 2012. 
[24] T. J. O'Shea, L.E. Ellison, and T. R. Stanley. Adult survival and population growth rate in colorado big brown bats (eptesicus fuscus). Journal of Mammalogy, 92(2):433$443,2011$.

[25] R Core Team. R: A Language and Environment for Statistical Computing. R Foundation for Statistical Computing, Vienna, Austria, 2013.

[26] M. Schaub, O. Gimenez, A. Sierro, and R. Arlettaz. Use of integrated modeling to enhance estimates of population dynamics obtained from limited data. Conservation Biology, 21:945-955, 2007.

[27] M. Scheffer. Critical Transitions in Nature and Society. Princeton University Press, Princeton, 2009.

[28] N. B. Simmons and G. F. Gunnell. Looking backwards: diversity in the bat fossil record. Bat Research News, 52:106, 2011.

[29] A.R. Sullivan, J.K. Bump, L.A. Kruger, and R. O. Peterson. Bat-cave catchment areas: using stable isotopes (deltad) to determine the probable origins of hibernating bats. Ecological applications, 22:1428, 2012.

[30] C. M. Taylor and D. R. Norris. Population dynamics in migratory networks. Theoreticla Ecology, 3:65-73, 2010.

[31] W.E. Thogmartin, R.A. King, P.C. McKann, J.A. Szymanski, and L. Pruitt. Population-level impact of White-nose Syndrome on the endangered Indiana bat. Journal of Mammalogy, 93:1086-1098, 2012.

[32] W.E. Thogmartin, C. A. Sanders-Reed, J. A. Szymanski, P. C. McKann, L. Pruitt, R. A. King, M. C. Runge, and R. E. Russell. White-nose syndrome is likely to extirpate the endangered Indiana bat over a large part of its range. Biological Conservation, 160:162-172, 2013.

[33] H. Wickham. ggplot2: Elegant Graphics For Data Analysis. Springer, New York, 2009.

[34] R. Wiederholt, L. López-Hoffman, J. Cline, R. A. Medellìn, P. Cryan, A. Russell, G. McCracken, J. Diffendorfer, and D. Semmens. Moving across the border: Modeling migratory bat populations. Ecosphere, 4:art114:http://dx.doi.org/10.1890/ES13-00023.1, 2013.

[35] K. Williams-Guillén, I. Perfecto, and J. Vandermeer. Bats limit insects in a neotropical agroforestry system. Science, 320:70, 2008. 